Ingeniería

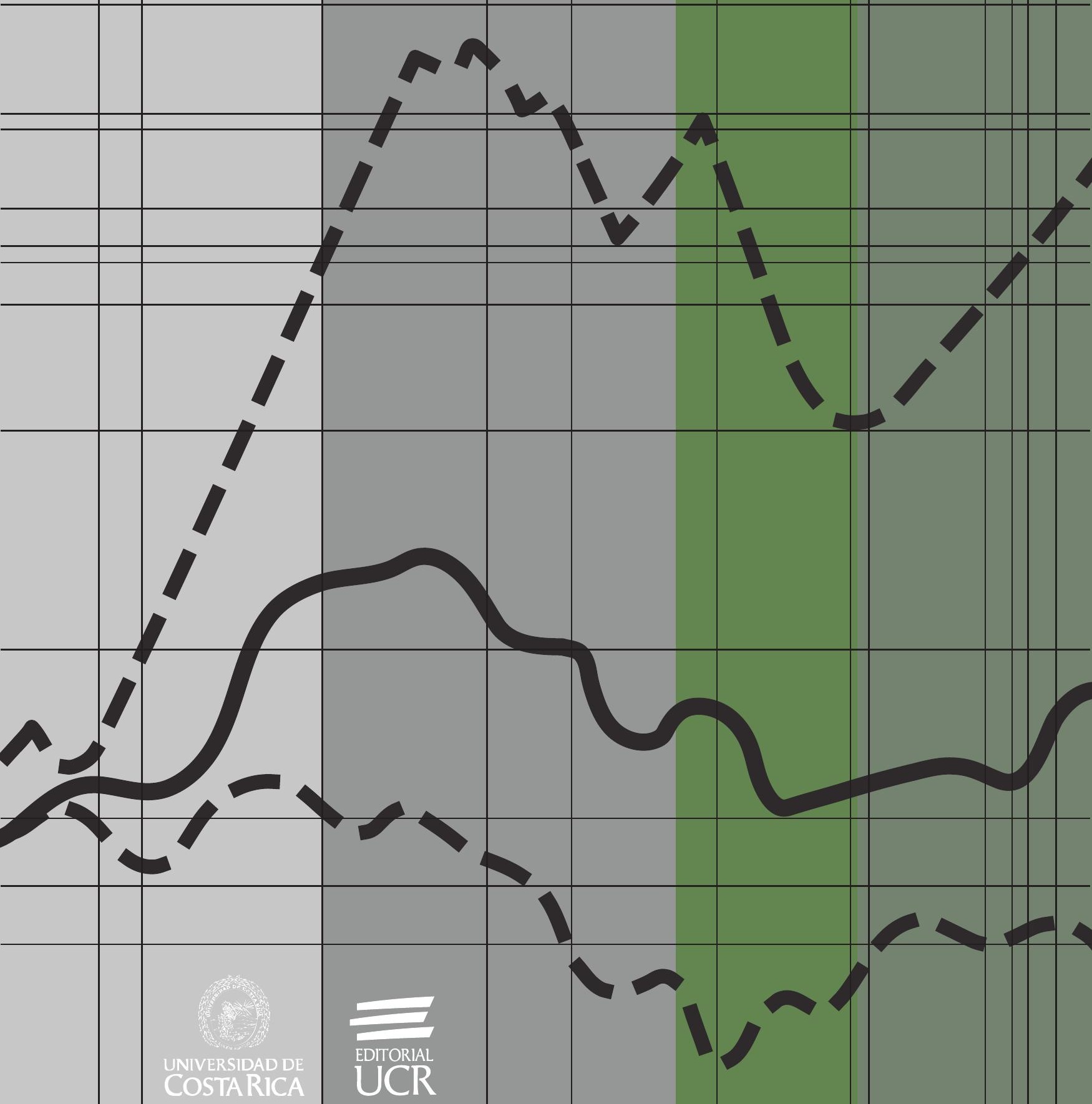




\title{
Empleo del método espectroscópico UV-Visible para determinación de asfaltenos en crudos extrapesados
}

\author{
Use of the Spectroscopy Method UV-Visible to Determination \\ of Asphaltene in Extra Heavy Crude Oils
}

\author{
Cesar Augusto Bernal Samaniego \\ Universidad de Panamá, NanoDispersions Technology Inc \\ cesarbernal10@gmail.com
}

Recibido: 24 enero 2018

Aceptado: 4 de junio 2018

\begin{abstract}
Resumen
En este estudio se comprobó que el método anteriormente estandarizado para la cuantificación de asfaltenos por espectroscopia UV-Visible en crudos pesados se puede emplear en crudos extrapesados. Al realizar las determinaciones se obtuvo una buena correlación y se muestra como una alternativa para la determinación de asfaltenos, ante el método tradicional ASTM 3279-07. Por lo tanto, se logra disminuir el tiempo y bajar el costo del análisis.
\end{abstract}

Palabras clave:

Cuantificación, hidrocarburos, petróleo, normas ASTM, desasfaltado.

\begin{abstract}
It was proved in this study that the previously standardized method for the asphaltenes' quantification by UV-Visible spectroscopy in heavy crudes can be used in extra-heavy crudes. A good correlation was obtained and it is shown as an alternative for the determination of asphaltenes, before the traditional method ASTM 3279-07. Therefore, it is possible to decrease the time and lower the cost of the analysis.
\end{abstract}

Key words:

Quantification, hydrocarbon, petroleum, ASTM rules, deasphalting. 


\section{INTRODUCCIÓN}

En los últimos años se ha visto un aumento en el consumo de energía, siendo aún la mayor fuente los derivados del petróleo. No obstante, se ha buscado explotar los yacimientos de crudos pesados y extrapesados como una fuente de energía alternativa, ya que representan la mayoría las reservas junto con el bitumen y se ha visto un declive en producción de crudos livianos o medianos (Diaz, Martínez, Patiño, \& Barrero, 2013).

El total de reservas de petróleo pesado, extrapesado y bitumen del mundo comprende un 70\% de las reservas mundiales. Por lo tanto, solamente el $30 \%$ es el llamado petróleo convencional (liviano o medio), como se puede observar en la figura 1. (Felix et al., 2006; Fergestad, 2016)

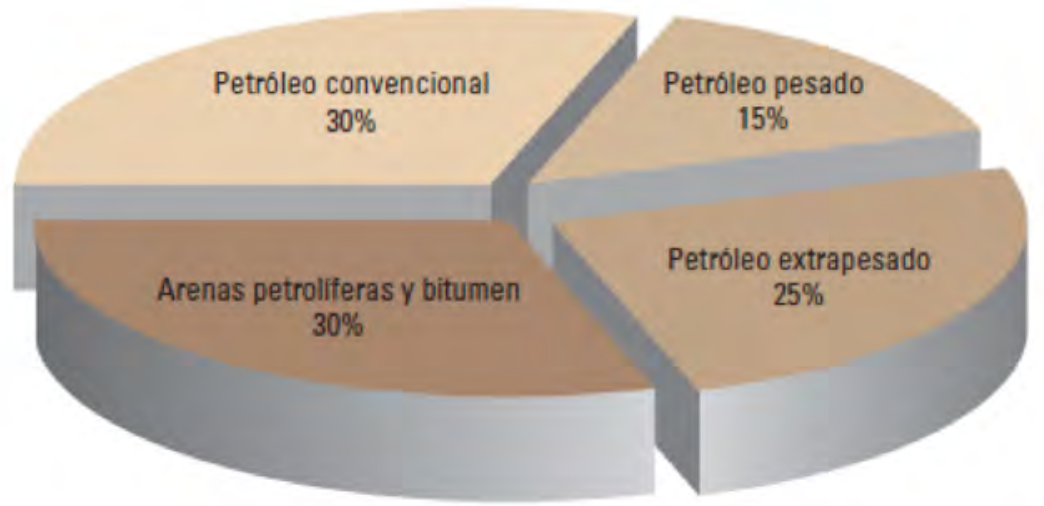

Figura 1. Total de reservas de petróleo en el mundo. Tomado de (Felix et al., 2006)

Sin embargo, los crudos pesados y extrapesados no eran vistos como fuente de energía debido a su composición, la cual presenta varias desventajas para trabajarlo. La principal es que poseen una alta viscosidad. Esta es ocasionada por las moléculas de hidrocarburos que son largas y complejas, con bajo contenido de hidrogeno en ellas, generando una alta fricción interna y por ende una alta viscosidad (Curtis et al., 2013; Grayson \& Hemingway, 2016).

La viscosidad dificulta no solo la extracción sino el transporte, aumentando como consecuencia el precio de producción de combustible a partir de él, ya que ocasiona sobrecostos en los procesos de extracción, transporte y refinación. La viscosidad de los petróleos convencionales puede oscilar entre 1 centipoise (cP), y aproximadamente 10 $\mathrm{cP}$, mientras que la viscosidad de los petróleos pesados y extrapesados puede fluctuar entre menos de $20 \mathrm{cP}$ y más de $1,000,000 \mathrm{cP}$ (Felix et al., 2006)

Una forma de caracterizar el tipo de crudo es la medida gravedad API (del inglés: American Petroleum Institute). Un valor mayor de 10 flot., Por tanto, mientras mayor sea la viscosidad del crudo, menor gravedad API; y a su vez también más difícil de 
extraer, transportar y refinar para la obtención de sus derivados. La clasificación se observa en la tabla 1.

Tabla 1. Clasificación de crudos de acuerdo a su gravedad API y su densidad. Tomado de (Chiriboga, 2007).

\begin{tabular}{lcr}
\hline Crudo & Escala API & Densidad $\left(\mathrm{g} / \mathrm{cm}^{3}\right)$ \\
\hline Ligero & $30-40^{\circ}$ & $0.87-0.83$ \\
Mediano & $22-29.9^{\circ}$ & $0.92-0.87$ \\
Pesado & $10-21.9^{\circ}$ & $1.00-0.92$ \\
Extrapesados & Menos $10^{\circ}$ & $>1.00$ \\
\hline
\end{tabular}

Otro aspecto que dificulta el empleo de estos crudos es la composición, pues presentan mayor cantidad de asfaltenos y resinas, así como baja proporción en los componentes de bajo peso molecular y fracciones livianas. También presentan mayor cantidad de azufre, nitrógeno, sales y metales pesados como el níquel y vanadio (Díaz et al., 2013).

Debido a la demanda de conseguir alternativas de fuentes de energía, se ha tenido un gran auge en el estudio de los crudos con mayor densidad y con ello la continua mejoración de métodos para facilitar el trabajo con estos, como lo es la diminución de tiempo y reactivos (Bernal, Castro, Ramos, \& Cárdenas, 2017).

El método tradicional para la cuantificación de asfaltenos ASTM 3279-07 requiere de mucho más tiempo para su medición: tiene una duración 2 días, mientras que él método espectroscópico dura 3h. Por otra parte, el método ASTM es más costoso, pues este requiere hasta 3 veces más de disolvente por muestra. Por esto la importancia de generar alternativas para su caracterización que mejoren el tiempo de procesado de las muestras y a su vez se pueda obtener un sistema de producción más eficiente, ya que este resultado es requerido para pasar a la segunda etapa de producción, pues cuando se trabaja con crudos se debe remover la mayor cantidad de asfaltenos posibles, con la finalidad de mejorar sus propiedades, como lo son disminuir la viscosidad y facilitar el transporte.

Cuando se realiza este proceso de desasfaltación se debe cuantificar la cantidad de asfaltenos remanentes en el producto desasfaltado o DAO, para su posterior utilización. No obstante, como se mencionó anteriormente, el método tradicional requiere de varios días para su determinación, ocasionando que haya atrasos en la producción. Es por ello que resulta de gran importancia el buscar alternativas de análisis que disminuyen el tiempo de cuantificación para que la producción no se detenga y funcione como un proceso continuo.

Por otra parte, es relevante estudiar si esta metodología se aplica a crudos extrapesados, ya que con anterioridad se había estandarizado para crudos pesados. Estos presentan similitudes en composición. Sin embargo, su viscosidad puede variar grandemente, ocasionando con ello que los crudos extrapesados sean de mayor dificultad para manipular. Por ende la importancia de estudiar el comportamiento con dicha método. 


\section{MÉTODOS Y MATERIALES}

\subsection{Materiales}

Los asfaltenos fueron obtenidos a partir del crudo colombiano rubiales, proporcionado por la empresa Pacific Rubiales Energy, el cual posee un contenido de 13,17 \% de asfaltenos. Este valor fue obtenido al hacer la cuantificación mediante el método ASTM D 3279-09. Los disolventes utilizados son marca Merck con un 99\% pureza. Para realizar las mediciones se empleó un espectrofotómetro UV-Visible modelo Cary 60 de Agilent Technologies con un rango de longitud de onda de 190 - $1100 \mathrm{~nm}$.

\subsection{Metodología}

Este trabajo busca correlacionar y reafirmar lo desarrollado en el estudio realizado anteriormente con los crudos pesados, utilizando los mismos métodos de precipitación de asfaltenos empleados en el estandarización descrita por (Bernal et al., 2017). Con el fin de poder crear una metodología para la cuantificación del contenido de asfaltenos en crudos extrapesados de una manera más rápida, con menos tiempo de análisis y uso de disolventes.

\section{Obtención de crudo desasfaltado y asfalteno}

Para la generación de muestras de crudo desasfaltado o DAO, con las cuales realizó la curva de calibración, se empleó el proceso de desasfaltado de crudo patentado por la empresa NanoDispersions Technology (Process of reducing viscosity of heavy crude oil by removal of asphaltene using a precipitating agent), denominado posteriormente CAR (Colloidal Asphaltene Removal), bajo la solicitud de patente número US20130264247A1.

En este proceso se obtienen tanto el crudo desasfaltado y como asfalteno con el cual se van a realizar las mezclas para la preparación de la curva de calibración.

\section{Curva de Calibración}

Posteriormente, se realizó un barrido de longitudes de onda a 200-800 nm para ver la relación de los asfaltenos purificados, el crudo extrapesado sin tratamiento y el crudo extrapesado desasfaltado. Al obtener una tendencia entre estas muestras se procede a realizar la curva de calibración.

Para el desarrollo de la curva de calibración, las correspondientes muestras obtenidas del proceso de obtención de crudo desasfaltado y asfaltenos fueron enviadas a un laboratorio externo para su análisis, con el fin de determinar la cantidad base de asfaltenos presente. Se obtuvo que en el DAO de crudo extrapesado se encontraba $0,6 \%$ de asfaltenos, mientras que para el crudo extrapesado sin tratamiento contenía un 13,2\% de asfaltenos. Las mezclas se preparan del mismo modo establecido en (Bernal et al., 2017). En la tabla 2 se encuentran los valores respectivos de cada reactivo para la elaboración de la curva de calibración.

Seguidamente, se realizaron las mediciones de absorbancia en función de la concentración del contenido de asfaltenos a las longitudes de onda de 600 y 700nm. 
Posteriormente, se procede a realizar el método de (Kharrat, Indo, \& Mostowfi, 2013) en donde se efectúa una diferencia entre las absorbancias obtenidas a dos diferentes longitudes de onda, con la finalidad de mejorar la linealidad y aumentar el coeficiente de correlación.

Tabla 2. Mezclas de crudo desasfaltado (DAO) y crudo extrapesado.

\begin{tabular}{ccc}
\hline \multicolumn{3}{c}{ Crudo Sintético extrapesado } \\
DAO $(\mathrm{g})$ & Crudo $(\mathrm{g})$ & $\begin{array}{c}\text { Porcentaje de } \\
\text { Asfaltenos }(\%)\end{array}$ \\
\hline 15,02 & $0 \mathrm{~g}$ & 0,6 \\
13,51 & 1,50 & 1,6 \\
12,01 & 3,01 & 2,6 \\
10,52 & 4,65 & 3,6 \\
9,03 & 5,99 & 4,6 \\
7,51 & 7,54 & 5,6 \\
6,01 & 9,00 & 6,6 \\
4,49 & 10,51 & 7,6 \\
3,06 & 12,02 & 8,6 \\
1,50 & 13.54 & 9.6 \\
\hline $0 \mathrm{~g}$ & 15.10 & 12 \\
\hline
\end{tabular}

Para la cuantificación de los porcentajes de asfaltenos en las muestras problema de crudo desasfaltado (DAO) (relaciones 1:1, 1:3), se analizaron en el UV-Visible y se midieron a longitudes de 600 y $700 \mathrm{~nm}$. Cada absorbancia fue correlacionada con los respectivos datos en la curva de calibración para obtener el correspondiente porcentaje de asfalteno.

\section{RESULTADOS Y DISCUSIÓN:}

Se llevó a cabo la determinación del contenido de asfaltenos para estos tipo de crudo, proponiendo utilizar el método espectroscópico UV-visible para la cuantificación de los asfaltenos en los procesos de obtención de DAOs con relación 1:1, 1:3 crudo:disolvente.

En este caso en particular trabajamos con el crudo extrapesado. En la generación de muestras de análisis se volvió a emplear el proceso CAR de desasfaltado de crudo. Una vez obtenido el DAO y los asfaltenos, se le realzaron sus debidos procesos de purificación. La figura 2 describe la correlación entre el crudo extrapesado sin tratamiento, los asfaltenos y DAO a través de un barrido de longitud de onda 200-800 nm a una concentración de 20 ppm. Los asfaltenos tienden a tener una absorbancia mayor que el crudo extrapesado sin tratamiento debido a su complejidad estructural, en comparación con el DAO, que presenta una disminución en la absorbancia con relación al crudo extrapesado sin tratar. 


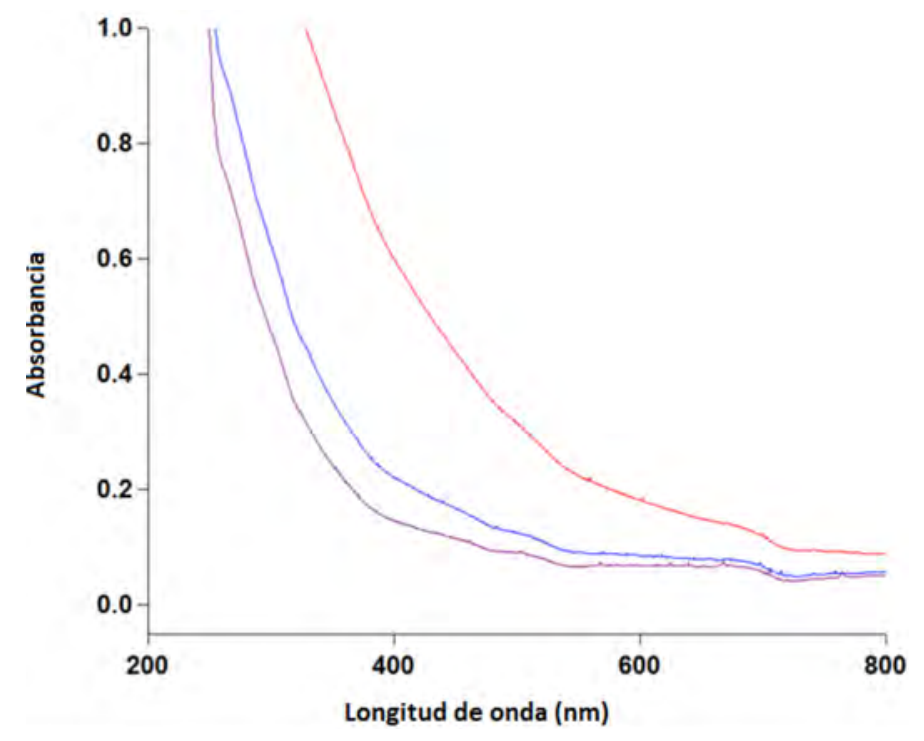

Figura 2. Barrido de longitud de 200 - $800 \mathrm{~nm}$ para crudo extrapesado (color azul), crudo extrapesado desasfaltado (color morado y asfaltenos (color rojo).

Posteriormente, se realizaron los barridos en el rango de longitudes de onda de 200$800 \mathrm{~nm}$ a las soluciones de crudos sintéticos mostradas en la tabla 2, donde se muestra la tendencia del aumento de la absorbancia con respecto al porcentaje de asfaltenos $(0,6$ a $12 \%$ ) en las mezclas generadas de crudos sintéticos extrapesados (figura 3 ).

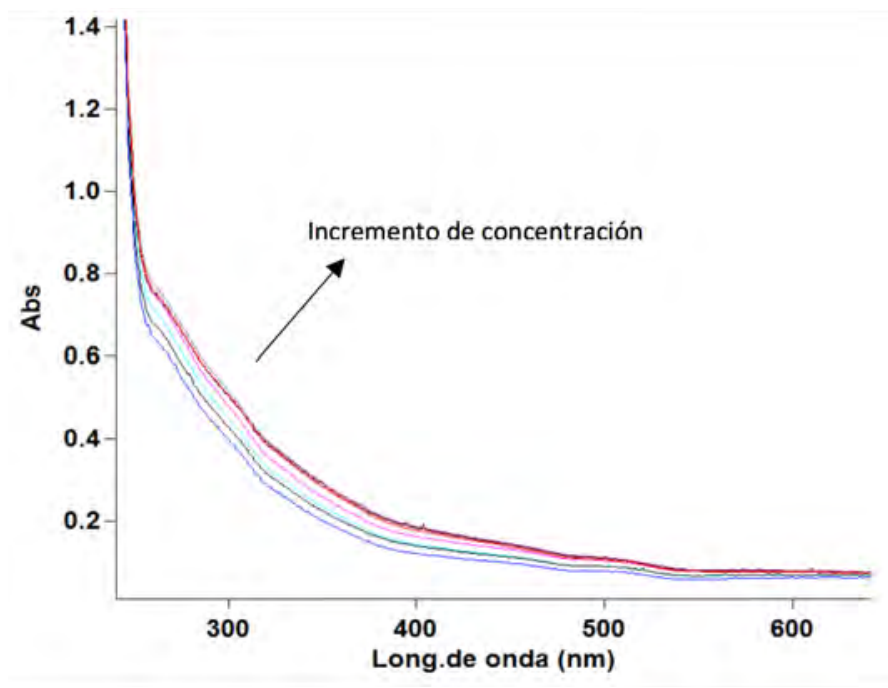

Figura 3. Curvas de barrido de longitud de ondas en el rango de $200 \mathrm{~nm}$ a $800 \mathrm{~nm}$ para mezclas de crudo extrapesado.

Posteriormente, se procede a realizar las curvas de calibración a diferentes longitudes de onda, obteniendo como mejor resultado las longitudes de 600 y $700 \mathrm{~nm}$ como lo muestra la figura 4 y la figura 5 . 
Mientras que, al realizar la correlación de longitudes de onda propuesta por Kharrat, Indo, \& Mostowfi, (2013) y Abdel, Kentaro, \& Farsshit, (2013) en la cual se establece hacer una diferencia en los datos de absorbancia a las dos longitudes de onda para cada concentración. Aplicando este método se obtuvieron las curvas que se muestran en la figura 6. Como resultado, se logró mejorar la correlación de los datos de la curva de calibración.

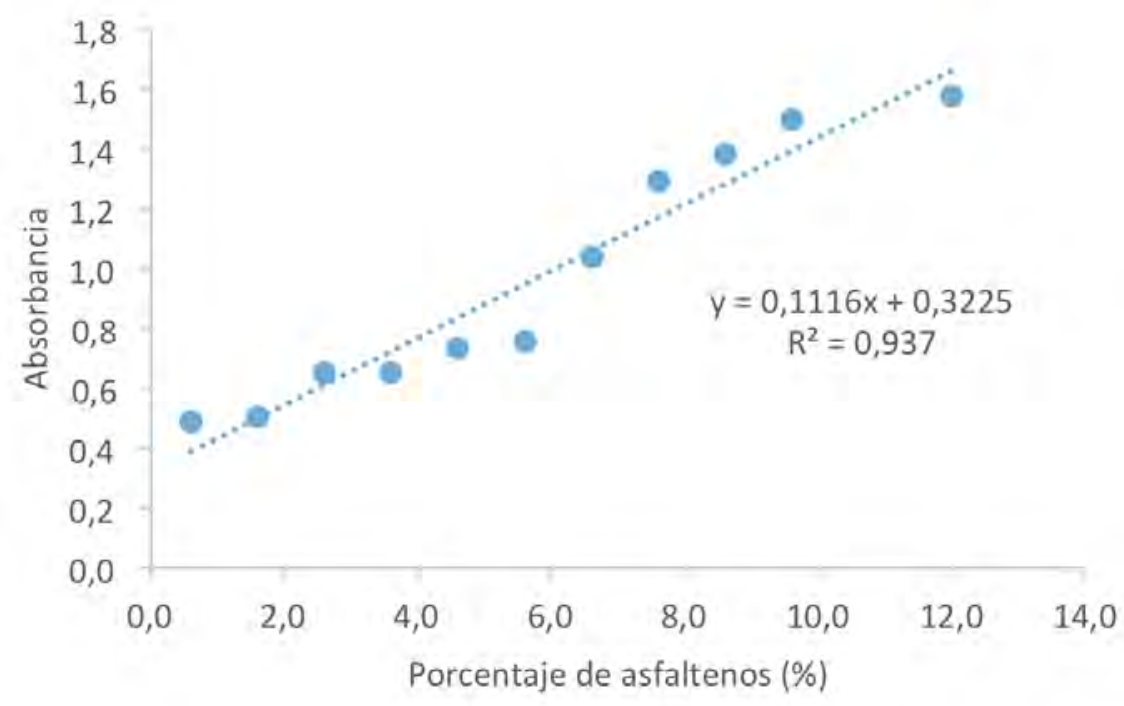

Figura 4. Absorbancias de los crudos sintéticos extrapesados 600nm.

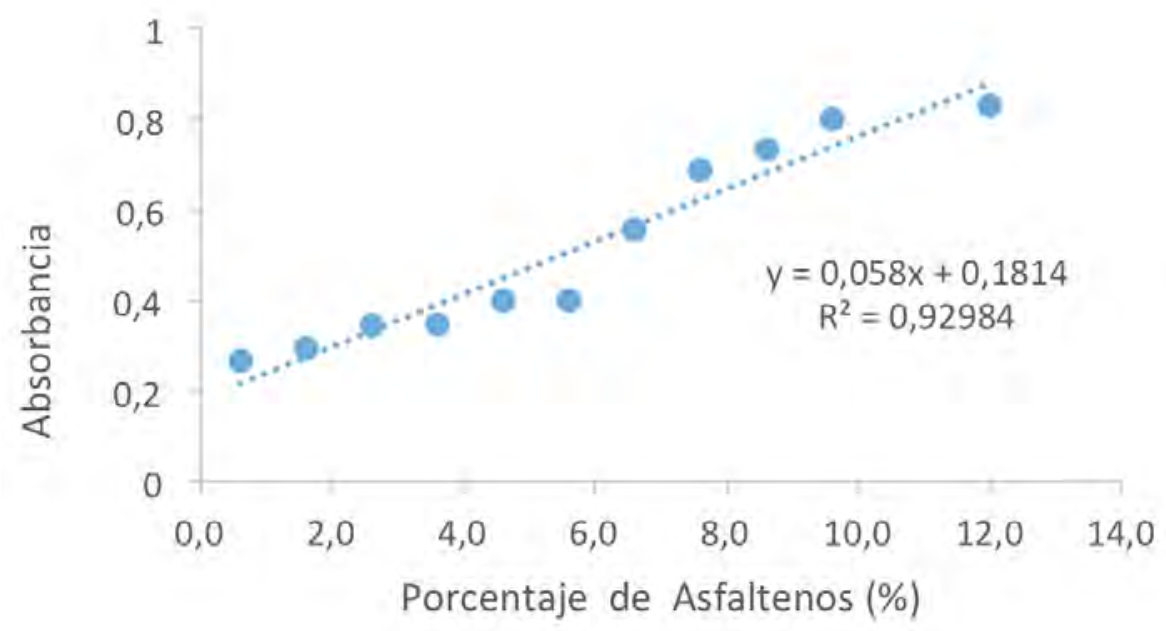

Figura 5. Absorbancias de los crudos sintéticos extrapesados 700nm. 


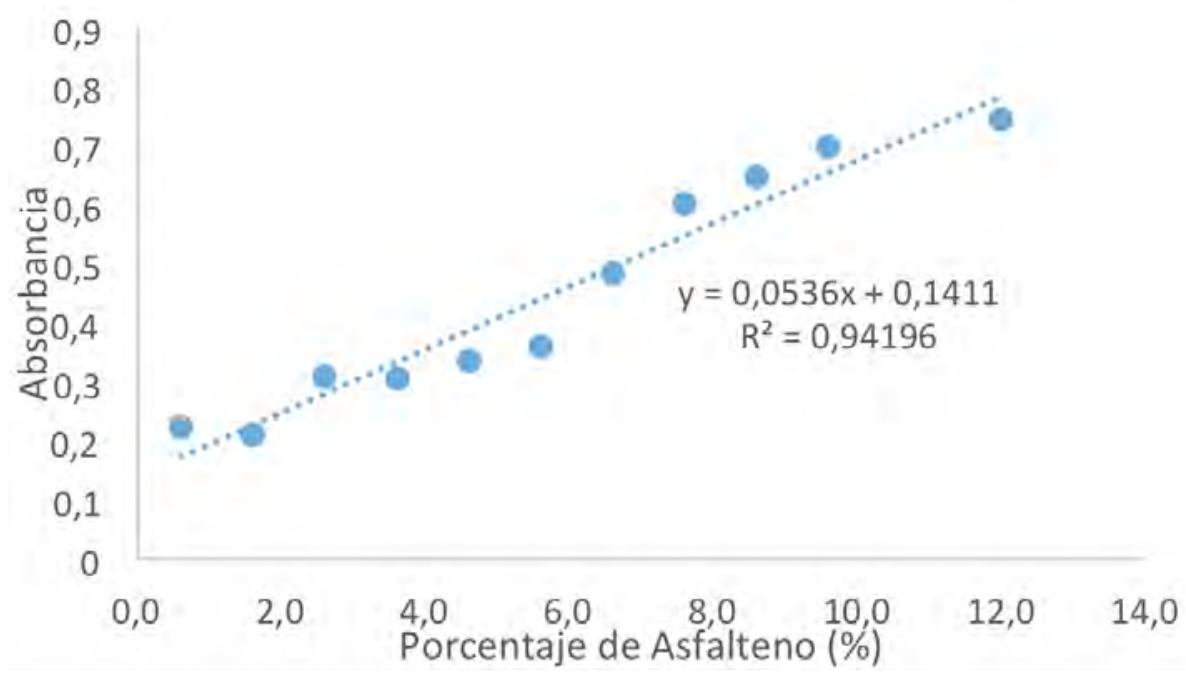

Figura 6. Diferencia de las curvas de absorbancia en función de la concentración de asfaltenos en la mezcla de Crudo sintético extrapesado y $600-700 \mathrm{~nm}$.

Una vez establecida la curva de calibración, se presentan los valores obtenidos por el método UV-visible para las muestras de crudo (relaciones 1:1, 1:3) utilizando el proceso de desafaltación CAR obteniendo los siguientes resultados que se muestran en la tabla 3. Al comparar estos datos con los proporcionados por la empresa del análisis externo se puede destacar que ambos presentan la misma tendencia. No obstante, con el método espectroscópico se obtienen valores menores que los de una norma ASTM. Esto se puede deber a las resinas que co-precipitan en la elaboración de esta metodología.

Tabla 3. Comparación entre los resultados obtenidos mediante el método UV- visible medido por la diferencia de absorbancias a las longitudes $\lambda=600-700 \mathrm{~nm}$ a las concentraciones en las relaciones $1: 1$ y $1: 3$ promedio.

\begin{tabular}{ccc}
\hline Relación de crudo:disolvente & $\begin{array}{c}\text { Porcentaje de asfaltenos en } \\
\text { UV-visible (\%) }\end{array}$ & $\begin{array}{c}\text { Porcentaje de asfaltenos indicado } \\
\text { por análisis externo con norma } \\
\text { ASTM }(\%)\end{array}$ \\
\hline $1: 1$ & $5,1 \pm 0,3$ & $6,6 \pm 0,5$ \\
$1: 3$ & $2,1 \pm 0,3$ & $4,4 \pm 0,5$ \\
\hline
\end{tabular}

Sin embargo, estos resultados demuestran que al mantener la tendencia y tener un porcentaje de error menor al 10\% (asumiendo como valor teórico el obtenido por la norma ASTM) se puede emplear como control en una planta de producción de derivados de petróleo con la finalidad de optimizar el proceso la cuantificación de asfaltenos en procesos de mejoramiento de crudo como lo es el desafaltado de crudo extrapesado. Logrando así disminuir los tiempos de análisis y uso excesivo de disolventes. 


\section{CONCLUSIONES}

El estudio realizado permite obtener las conclusiones siguientes:

- Es posible medir la cantidad de asfaltenos del crudo Rubiales utilizando un método espectroscópico con buena precisión. Las curvas obtenidas en longitudes de onda de 600 y $700 \mathrm{~nm}$ permiten obtener un coeficiente de correlación superior al 0,90.

- El uso del método que considera la diferencia de longitudes de onda también permite obtener buenos resultados, mejorado la correlación de los datos obtenidos con la medición de una longitud de onda.

\section{REFERENCIAS}

Abdel, K., Kentaro, I., \& Farsshit, M. (2013). Asphaltene content measurement using an opticalspectroscopy. Energy Fuels.

Bernal, C., Castro, A., Ramos, M., \& Cárdenas, A. (2017). Estandarización del método espectroscópico UV-Visible para la determinación del contenido de asfaltenos en crudos pesados. Revista de Ingeniería, 27(2), 1-13.

Chiriboga, J. A. (2007). Propuesta de explotación y manejo de crudos pesados petroproducción, campo Oglan. Instituto de altos estudios nacionales.

Curtis, C., Guzmán-garcía, A., Huggins, C., Knauer, L., Minner, M., \& Rough, H. (2013). Yacimientos de petróleo pesado. Oilfield Review, 12(6), 32-55.

Diaz, J. C., Martínez, R., Patiño, E. J., \& Barrero, R. (2013). Estudio experimental sobre la eficiencia de un tratamiento de ultrasonido en un sistema de flujo continuo para la reducción de viscosidad de crudo pesado. Revista ION, 26(2), 47-63.

Felix, J., Badry, B., Bremner, C., Brough, B., Steates, C., Baker, A., ... Cárdenas, J. (2006). La importancia del petróleo pesado. Oilfield Review, 18(3), 38-59.

Fergestad, I. M. (2016). Petróleo Pesado. Oilfield Review, 2-4.

Grayson, S., \& Hemingway, J. (2016). Evaluación temprana de la movilidad de los petróleos pesados. Oilfield Review, 28(1), 4-9.

Kharrat, A. M., Indo, K., \& Mostowfi, F. (2013). Asphaltene Content Measurement Using an Optical Spectroscopy Technique. Energy \& Fuels, 27(5), 2452-2457. https://doi.org/10.1021/ ef $400050 \mathrm{y}$ 